\title{
Modified spontaneous emission from a two- dimensional photonic bandgap crystal slab
}

\author{
Reginald K. Lee, Yong Xu, and Amnon Yariv \\ Applied Physics and Electrical Engineering, California Institute of Technology, 1200 E. California Boulevard, \\ Pasadena, California 91125
}

Received February 14, 2000

\begin{abstract}
A two-dimensional photonic crystal patterned into a thin dielectric slab waveguide is shown to alter drastically the lifetime of spontaneous emission as well as the radiation pattern. This means that although the light extraction efficiency can be greatly enhanced, inhibited spontaneous emission within the photonic bandgap can result in low power output from such a structure. Strongly inhibited emission is found within the photonic bandgap as well as enhanced emission into the conduction band modes for certain geometries. Coupled with enhanced extraction efficiency in the photonic conduction band, this results in the possibility of a structure with increased total power efficiency. (c) 2000 Optical Society of America [S0740-3224(00)01008-0]

OCIS codes: 270.5580, 230.3990, 230.3670, 130.3130, 020.5580.
\end{abstract}

\section{INTRODUCTION}

Spontaneous emission from a radiating system can be significantly increased ${ }^{1}$ or inhibited $^{2}$ in a resonant cavity. Photonic bandgap structures in light-emitting materials have been predicted to be able to exhibit enhanced or inhibited spontaneous emission., ${ }^{3,4}$ The possibility of control over the fundamental spontaneous-emission properties has resulted in a great deal of recent interest.

One possible application of photonic crystals is the light-emitting diode (LED). The external efficiency of LED's is typically relatively low, primarily owing to low extraction efficiency of the spontaneously emitted radiation because of total internal reflection. Many approaches have been proposed to increase this extraction efficiency by enlarging the photon escape cone or using photon recycling. ${ }^{5}$ The fundamental spontaneousemission properties of the device can also be modified, for example, by use of a Fabry-Perot microcavity. ${ }^{6}$ However, these resonant cavity LED's modify the emission in only a narrow spectral range.

Inhibited spontaneous emission in photonic crystals has been demonstrated for microwave radiation by Yablonovitch and Gmitter ${ }^{7}$ using a period structure composed of low-loss dielectric material. However, measurements in the optical regime with solid-state light-emitter photonic crystals have been hampered by the difficulty in fabrication of such nanoscale structures. A full threedimensional photonic bandgap can be achieved when the dielectric contrast between the scattering centers and the surrounding medium exceeds a critical value of $\epsilon_{r}>10$. Although the dielectric constant is weaker than this criteria $\left(\epsilon_{r}=1.45\right)$, Martorell and Lawandy ${ }^{8}$ have observed inhibited spontaneous emission in an ordered aqueous suspension of polystyrene spheres even though their structure did not exhibit a complete bandgap. More recently, Petrov et al. ${ }^{9,10}$ have observed modified spontaneous spectra and decay kinetics of dye molecules in an artificial opal photonic crystal.
In this paper we show that the in-plane bandgap for a finite two-dimensional photonic crystal slab can be used to achieve an inhibition in the spontaneous-emission rate by an order of magnitude even though it lacks a complete photonic bandgap. The effect of the dipole position in the photonic lattice on the emission rate is briefly considered. The combination of changes in the emission pattern and the emission rate can result in an enhancement or inhibition of the external quantum efficiency.

\section{RESULTS AND DISCUSSION}

\section{A. Dipole Position Dependence}

Recently, ${ }^{11}$ a new approach to modify the spontaneous emission and increase significantly the extraction efficiency in LED's over a wide frequency range with a thin slab of two-dimensional photonic crystal was proposed. However, only the modification to the emission pattern was considered. In a light-emitting structure with residual nonradiative recombination, it is also necessary to consider the radiation rate. In the case in which the extraction efficiency is strongly enhanced, if the radiation rate is correspondingly inhibited, the resulting net extracted power may be enhanced or reduced, depending on the associated nonradiative recombination rate.

Spontaneous emission in a two-dimensional photonic bandgap structure has been theoretically examined for a honeycomb structure ${ }^{12}$ as well as the two-dimensional triangular array of holes. ${ }^{13}$ However, this previous analysis of emission-rate suppression in photonic crystals estimates only the solid angle fraction that can be affected by the photonic crystal. A more precise evaluation requires a self-consistent analysis that takes into account the interaction between the light source and the photonic bands as well as the finite size of the crystal in the third dimension. Photonic bandgap materials can completely suppress spontaneous emission within the gap region. ${ }^{3}$ However, the radiation dynamics depend on the local density of states within the region containing radiating spe- 
cies rather than the total free-space density of states outside the crystal. The result is that radiation within a finite photonic structure can be completely controlled. ${ }^{14}$

We have previously proposed and demonstrated a finite-difference time-domain method for direct calculation of the spontaneous-emission rate in an arbitrary dielectric structure. ${ }^{15}$ Recently, Hwang et al. ${ }^{16}$ have independently developed similar calculations of spontaneousemission rate in various photonic crystal structures using a similar finite-difference time-domain method. Although this method is generally useful in being able to deal with arbitrary structures and inherently includes all localized, guided, and extended modes, careful consideration must be given to the position dependence of the emission rate on the dipole source location. The choice of regions for the calculation of the local density of states can have a significant effect on the resulting calculated radiation rate. Therefore, the single-point dipole method used by Hwang et al. ${ }^{16}$ to calculate the spontaneousemission rate will depend on the choice of dipole position. To overcome this difficulty, an average over possible dipole positions is necessary to determine the spontaneousemission properties of the structure as a whole. Figure 1 shows an example of single-dipole-calculated spontaneous-emission rates at various dipole positions by use of a method similar to that used by Hwang et $a l .{ }^{16}$ for a triangular lattice of holes with $r / a=0.32$ in a slab with thickness $d / a=0.375$. Figure 1 (a) shows a schematic of the structure with three dipole locations indicated that

(a)

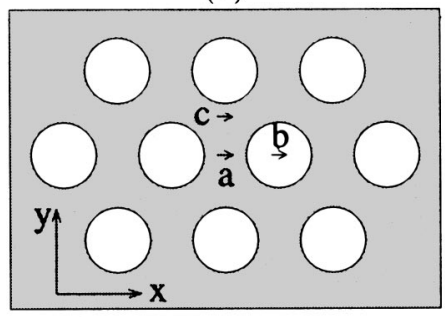

(b)

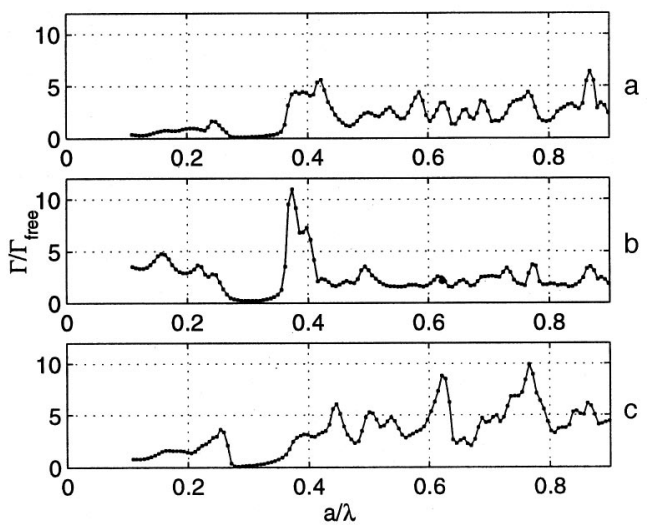

Fig. 1. Position dependence of the spontaneous-emission rate by use of a single-point dipole at different locations in the photonic crystal structure. (a) Schematic indicating the calculated dipole locations in the triangular lattice. (b) Spontaneous-emissionrate spectrum relative to the free-space rate for the different dipole locations. correspond to the three spectra shown in Fig. 1(b). As expected, the emission-rate spectrum can clearly be changed dramatically by the choice of dipole position within the photonic lattice. In all three cases, the bandgap region can be seen approximately in the frequency range 0.27 to 0.36 .

In the Coulomb gauge, the transverse field must satisfy the following wave equation in the presence of a dipole source:

$$
\Delta^{2} \mathbf{E}-\epsilon(\mathbf{x}) \mu_{0} \frac{\partial^{2} \mathbf{E}}{\partial t^{2}}=\mu_{0} \frac{\partial^{2} \mathbf{P}}{\partial t^{2}},
$$

where the polarization is assumed to be a point dipole oscillator located at $\mathbf{x}_{0}$. In

$$
\mathbf{P}(\mathbf{x}, t)=d(\mathbf{t}) \delta\left(\mathbf{x}-\mathbf{x}_{0}\right),
$$

$\epsilon(\mathbf{x})$ is the position-dependent permittivity and $\mu_{0}$ is the permeability that is assumed to be spatially invariant. We have shown previously ${ }^{15,17}$ that the classical dipole radiation power in a finite-difference time-domain calculation can be used to determine the quantum mechanical spontaneous lifetime as

$$
\left[\frac{P^{\text {cav }}}{P_{\text {vac }}}\right]_{\text {quantum }}=\left[\frac{P^{\text {cav }}}{P^{\text {vac }}}\right]_{\text {classical }}=\frac{\tau_{\text {vac }}}{\tau_{\text {cav }}},
$$

where cav denotes values for the cavity structure and vac denotes values calculated for a vacuum. We use a fully vectorial three-dimensional calculation with perfectly matched layer absorbing boundary conditions ${ }^{18}$ to directly solve Eq. 1. A large number of point dipoles with random polarization, Lorentzian line shape, and Poisson distributed in time are used as radiation sources to average out dependencies on polarization and position within the lattice. Only dipoles polarized in the plane of the slab are considered, since they correspond to emission from semiconductor quantum wells. The dipole sources are also limited to the regions of high dielectric constant so that there are no radiation sources in the air regions. The single-dipole calculations used broadband Gaussian pulse excitation analyzed by discrete Fourier transformation. ${ }^{19}$ The multiple-dipole-averaged simulations used narrowband Lorentzian line-shape dipoles and the total timeaveraged power flow. This is equivalent to averaging the frequency response over the dipole homogeneous linewidth.

Owing to computational time restrictions, a small $6 \times 6$ photonic crystal unit cell domain was used. For comparison, an $8 \times 8$ cell domain was calculated, and these results were not qualitatively different from the smaller calculations. The structure studied in this paper is shown schematically in Fig. 2 (inset). We define the extraction efficiency, $\eta_{\text {ext }}$ as the ratio of power through the top surface of the domain $(+z)$ to the total power. We also introduce a detection efficiency, $\eta_{\text {det }}$ to characterize the power that might be practically collected normal to the slab. This is defined as the power emitted through a square surface parallel to the slab where the sides of the square subtend a half-angle of $\sim 52^{\circ}$ (a collection cone with this half-angle corresponds to a numerical aperture of $\mathrm{NA} \approx 0.8$ ). 
(a)

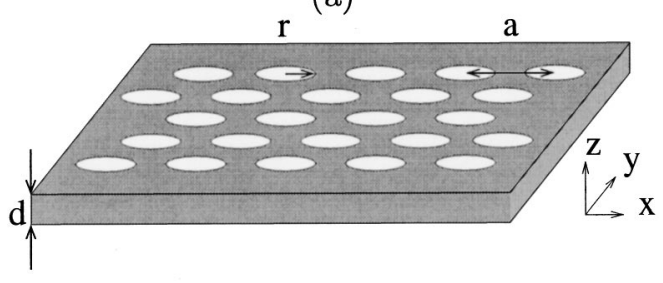

(b)

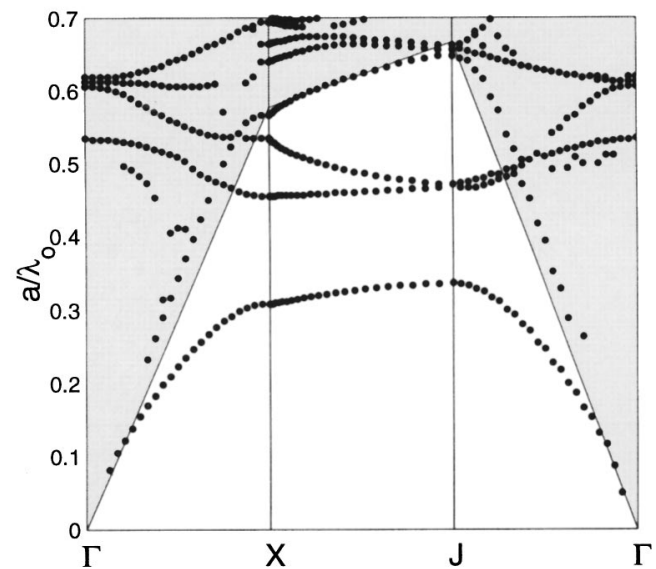

Fig. 2. (a) Schematic of the triangular lattice photonic crystal slab. (b) Calculated in-plane bandstructure with $d / \lambda=0.25$ and $r / a=0.35$ for TE-like modes. The shaded region corresponds to the unguided (extended) modes.

\section{B. Fixed $d / a$ Structure}

We first consider a photonic crystal slab structure with fixed waveguide thickness to lattice parameter ratio, $d / a$. The band structure for in-plane wave vectors for this structure is shown in Fig. 2 by use of a finite-difference time-domain method similar to that of Painter et al. ${ }^{20}$ Figure 3 shows the corresponding spontaneous-emissioncalculation results. The left plot shows the spontaneousemission rate $\left(\Gamma_{\mathrm{sp}}\right)$ relative to the value in free space. At low frequencies $(a / \lambda<0.2)$, the spontaneous-emission rate is much lower than the free-space value. Since the wavelength is much larger than the lattice spacing, an effective index approximation can be used that implies that the emission rate will scale roughly as the effective index and the waveguide thickness, $d / \lambda$. The emission rate within the in-plane bandgap $(a / \lambda \approx 0.3-0.4)$ shows a suppression by as much as $3 \times$. Above the upper band edge, there is a strong enhancement of the emission rate to as much as $8 \times$ the free-space rate, corresponding to emission into the photonic conduction bands. Broad peaks in the spontaneous-emission rate in the frequency range $0.42-0.57$ and centered at $\sim 0.65$ correspond respectively to the first two guided modes of the conduction band in Fig. 2 and the band that lies just below the light line in the $\mathbf{X J}$ direction in Fig. 2.

The extraction and detection efficiencies $\eta_{\text {ext }}$ and $\eta_{\text {det }}$ are shown in Fig. 3 (center). The total extraction efficiency rises to nearly 50\% (complete extraction) in the bandgap region, along with an increase in the detection efficiency $(\sim 41 \%)$. This agrees quite well with the results of Fan et $a l .{ }^{11}$ and is attributed to the photonic crystal inhibiting guided modes within the slab. However, for frequencies above the band edge, both the extraction and detection efficiencies are significantly lower (25 and $10 \%$, respectively). An increase in the extraction efficiency also occurs for frequencies above $\sim 0.65$, corresponding to crossing the light line to the unguided modes.

Although there are regions of nearly complete light extraction, in practice, this is not necessarily useful because of the greatly reduced emission rate. In a medium with nonradiative decay, the reduced emission rate can result in a corresponding reduction in the internal quantum (radiative) efficiency $\left[\eta_{\mathrm{rad}}=\Gamma_{\mathrm{rad}} /\left(\Gamma_{\mathrm{rad}}+\Gamma_{\mathrm{nr}}\right)\right]$. If, for example, a material has a $\eta_{\text {rad }}=90 \%$ in bulk form, the $3 \times$ reduction in the spontaneous-emission rate results in $\eta_{\mathrm{rad}}$ being reduced to $\sim 75 \%$, assuming a fixed nonradiative rate. A better comparison may be made by considering the emission rate through a surface of interest, the extraction rate $\Gamma_{\text {ext }}$ for the infinite $x y$ plane or the detection rate $\Gamma_{\text {det }}$ for the previously defined detection surface. This is shown in Fig. 3 (right), normalized to the corresponding rates for free space. The extraction and detection rate curves are very similar, owing to the similarity in $\eta_{\text {ext }}$ and $\eta_{\text {det }}$. Above the bandgap, the emission rate is enhanced enough to compensate for the lower extraction efficiency, resulting in as much as $\sim 10 \times$ enhancement of $\Gamma_{\text {ext }}$ and $\Gamma_{\operatorname{det}}$ at $a / \lambda \sim 0.53$. In the bandgap frequency region and below, the extraction rate is much smaller than the free-space rate. In the bandgap region, although the $\eta_{\text {ext }}$ is high, the corresponding emission rate $\Gamma_{\mathrm{sp}}$ is low.

\section{Fixed $d / \lambda$ Structure}

A structure consisting of a slab of photonic crystal in which the emission wavelength $(\lambda)$ and slab thickness $(d)$ are fixed and the lattice spacing $(a)$ is varied is of interest owing to the ease with which the lattice spacing can be easily varied lithographically. ${ }^{21}$ In this case, by increasing the normalized frequency $(a / \lambda)$, we decrease the waveguide thickness $d / a$. This results in a shift of the bandgap toward higher frequencies, ${ }^{20}$ as shown in Fig. 4. Since experimental measurements ${ }^{22-24}$ are typically un-

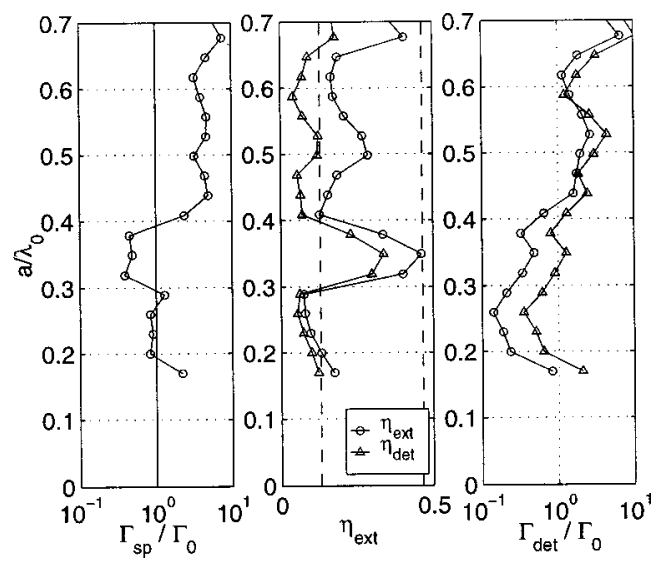

Fig. 3. Spontaneous emission from a photonic crystal slab with $d / a$ constant. Left, spontaneous-emission rate normalized to the free-space rate (logarithmic scale). Center, extraction (circles) and detection (triangles) efficiencies as defined in the text. The values for an unpatterned slab are indicated by the dashed line. Right, detection (triangles) and extraction (circles) rates (logarithmic scale) from a photonic crystal slab normalized to the rate for free space. 


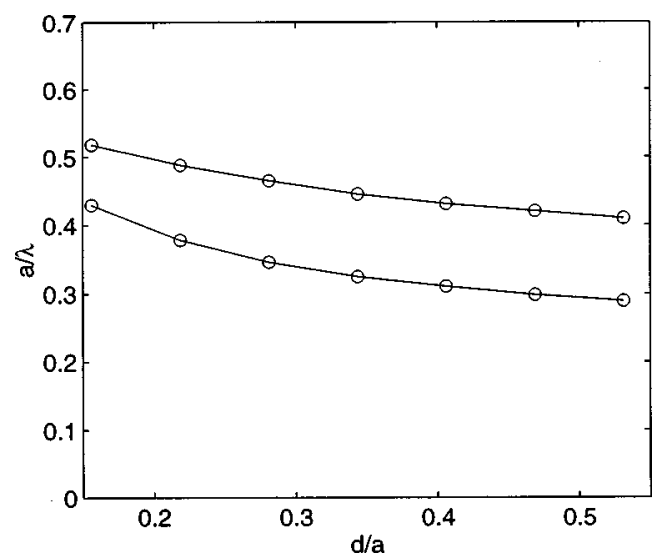

Fig. 4. Variation in band-edge frequencies as a function of waveguide thickness $d / a$ for a triangular lattice of holes with constant $r / a=0.35$, calculated with a three-dimensional finitedifference time-domain method.

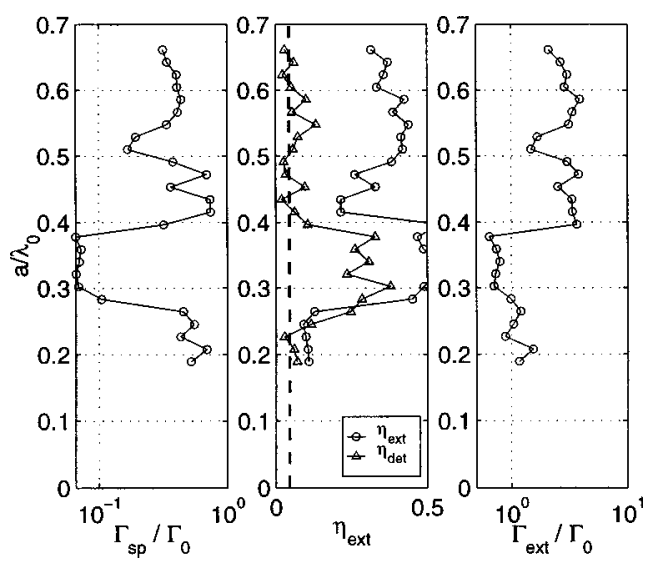

Fig. 5. Power extraction from a photonic crystal slab with $d / \lambda$ constant. Left, spontaneous-emission rate (logarithmic scale) normalized to the value in a slab waveguide. Center, extraction (circle) and detection (triangle) efficiencies. The corresponding values for an unpatterned slab are indicated by the dashed line. Right, extraction rate (logarithmic scale) from a photonic crystal slab normalized to the rate for an unpatterned slab with the same thickness $d / \lambda$.

der conditions of constant thickness $(d / \lambda)$, the calculations for constant $d / a^{11,16}$ cannot be directly compared. The emission rate, extraction and detection efficiencies, and the detection rate are shown in Fig. 5. In this case the emission rates have been normalized to the corresponding slab waveguide rate instead of the free-space rate. The strong suppression of the spontaneousemission rate by approximately $10 \times$ in the bandgap frequency range is again seen with the corresponding sharp increase in both the extraction and the detection efficiencies such that nearly all emitted photons are extracted normal to the slab. However, above the bandgap region, there is no dramatic enhancement of $\Gamma_{\mathrm{sp}}$ and for $a / \lambda>0.5, \Gamma_{\mathrm{sp}}$ is in fact inhibited by approximately $2 \times$. Since the waveguide to wavelength ratio $(d / \lambda)$ is fixed, the aforementioned bandgap shift upward in frequency results in the conduction bands moving toward the light line. The field confinement is thereby reduced, decreasing the enhancement of $\Gamma_{\mathrm{sp}}$ [Fig. 5 (left)]. Also, the re- duced vertical confinement by the slab index guiding results in a slightly higher extraction and detection efficiency in the conduction bands [Fig. 5 (center) $\sim 40$ and $\sim 15 \%$ versus 25 and $10 \%$ in the previous case].

The extraction rate for $d / \lambda$ fixed is shown in Fig. 5 (right). Below the upper band edge, $\Gamma_{\text {ext }}$ remains similar to the slab extraction rate. This suggests that the inhibition that occurs within the bandgap is mostly due to the solid angle fraction affected by the photonic structure. $\Gamma_{\text {ext }}$ is directly related to the coupling of the dipoles to the unguided radiation modes so that the inhibition of guided modes does not strongly affect the extraction rate. Above the bandgap, there is a combination of enhanced extraction efficiency and only slight inhibition of $\Gamma_{\mathrm{sp}}$, resulting in a $\sim 5 \times$ enhancement of the extraction rate. For a medium with nonradiative decay, similar behavior would be expected from a measurement of emitted power with increased power output above the upper band edge in the $+z$ direction. Previously, spontaneous-emission-power measurements from similar triangular lattice photonic crystal slabs have been presented ${ }^{22,23}$ that showed strongly enhanced power extraction $(\sim 10 \times)$ above the upper band edge. This qualitative behavior for a triangular lattice of air holes corresponds well with Fig. 5 (right), which shows a large power enhancement for frequencies above the bandgap over those below or within the gap region. Strong enhancement of the extraction efficiency from a hexagonal lattice of semiconductor microcolumns has also been observed by Baba et al. ${ }^{24}$ but without a corresponding enhancement of the power extraction. For this geometry, there was no measurable change in the spontaneous lifetime. ${ }^{24}$ However, the power extraction in the presence of nonradiative recombination mechanisms depends on both the spontaneous emission rate and the extraction efficiency.

\section{CONCLUSION}

We have shown that a two-dimensional photonic bandgap structure in a thin slab can strongly suppress or enhance the spontaneous-emission rate by approximately one order of magnitude even though this structure lacks a complete three-dimensional bandgap. The large enhancement rates at the band edges predicted in single-dipole calculations are strongly dependent on the choice of dipole position, so that spatial averaging is necessary to estimate the general emission properties of the photonic structure as a whole. The bandgap structure can also be used to enhance the vertical extraction efficiency. Emission into the conduction bands can be enhanced while relatively large extraction efficiency is maintained. In the presence of nonradiative processes, this could result in a structure with enhanced internal and external quantum efficiencies.

\section{ACKNOWLEDGMENTS}

This work was supported by the U.S. Army Research Office, the U.S. Office of Naval Research, and the Defense Advanced Research Projects Agency. R. Lee is supported by the National Science and Engineering Research Council of Canada. 


\section{REFERENCES}

1. E. Purcell, "Spontaneous emission probabilities at radio frequencies," Phys. Rev. 69, 681 (1946).

2. D. Kleppner, "Inhibited spontaneous emission," Phys. Rev. Lett. 47, 233-236 (1981).

3. E. Yablonovitch, "Inhibited spontaneous emission in solidstate physics and electronics," Phys. Rev. Lett. 58, 2059 2062 (1987)

4. S. John, "Strong localization of photons in certain disordered dielectric superlattices," Phys. Rev. Lett. 58, 2486 2489 (1987)

5. I. Schnitzer, E. Yablonovitch, C. Caneau, T. Gmitter, and A. Scherer, "30-percent external quantum efficiency from surface textured, thin-film light-emitting diodes," Appl. Phys. Lett. 63, 2174-2176 (1993).

6. E. Schubert, Y. Wang, A. Cho, L. Tu, and G. Zidzik, "Resonant cavity light-emitting diode," Appl. Phys. Lett. 60, 921923 (1992).

7. E. Yablonovitch and T. Gmitter, "Photonic band structure: the face-centered-cubic case," Phys. Rev. Lett. 63, 19501953 (1989).

8. J. Martorell and N. Lawandy, "Observation of inhibited spontaneous emission in a periodic dielectric structure," Phys. Rev. Lett. 65, 1877-1800 (1990).

9. E. Petrov, V. Bogomolov, I. Kalosha, and S. Gaponenko, "Spontaneous emission of organic molecules embedded in a photonic crystal," Phys. Rev. Lett. 81, 77-80 (1998).

10. S. Gaponenko, V. Bogomolov, E. Petrov, A. Kapitonov, D. Yarotsky, I. Kalosha, A. Eychmueller, A. Rogach, J. McGilp, U. Woggon, and F. Gindele, "Spontaneous emission of dye molecules, semiconductor nanocrystals, and rare-earth ions in opal-based photonic crystals," J. Lightwave Technol. 17, 2128-2137 (1999)

11. S. Fan, P. Villeneuve, J. Joannopoulos, and E. Schubert, "High extraction efficiency of spontaneous emission from slabs of photonic crystals," Phys. Rev. Lett. 78, 3294-3297 (1997).

12. T. Søndergaard, J. Broeng, A. Bjarklev, K. Dridi, and S. Barkou, "Suppression of spontaneous emission for a twodimensional honeycomb photonic bandgap structure esti- mated using a new effective-index model," IEEE J. Quantum Electron. 34, 2308-2313 (1998).

13. X. Feng and Y. Arakawa, "Off-plane angle dependence of photonic band gap in a two-dimensional photonic crystal," IEEE J. Quantum Electron. 32, 535-542 (1996).

14. S. John and K. Busch, "Photonic bandgap formation and tunability in certain self-organizing systems," J. Lightwave Technol. 17, 1931-1943 (1999).

15. Y. Xu, J. Vuckovic, R. Lee, O. Painter, A. Scherer, and A Yariv, "Finite-difference time-domain calculation of spontaneous emission lifetime in a microcavity," J. Opt. Soc. Am. B 16, 465-474 (1999).

16. J. Hwang, H. Ryu, and Y. Lee, "Spontaneous emission rate of an electric dipole in a general microcavity," Phys. Rev. B 60, 4688-4695 (1999).

17. Y. Xu, R. Lee, and A. Yariv, "Quantum analysis and the classical analysis of spontaneous emission in a microcavity," Phys. Rev. A 61, Art. No. 33807 (2000).

18. J. Berenger, "A perfectly matched layer for the absorption of electromagnetic waves," J. Comput. Phys. 114, 185-200 (1994)

19. C. Furse and O. Gandhi, "Why the DFT is faster than the FFT for FDTD time-to-frequency domain conversions," IEEE Microwave Guid. Wave Lett. 5, 326-328 (1995).

20. O. Painter, J. Vuckovic, and A. Scherer, "Defect modes of a two-dimensional photonic crystal in an optically thin dielectric slab," J. Opt. Soc. Am. B 16, 275-285 (1999).

21. C. Cheng, V. Arbet-Engels, E. Yablonovitch, and A. Scherer, "Lithographic band gap tuning in photonic band gap crystals," J. Vac. Sci. Technol. B 14, 4110-4114 (1996).

22. A. Scherer, O. Painter, B. D'Urso, R. Lee, and A. Yariv, "InGaAsP photonic band gap crystal membrane microresonators,” J. Vac. Sci. Technol. B 16, 3906-3910 (1998).

23. M. Boroditsky, R. Vrijen, T. Krauss, R. Coccioli, R. Bhat and E. Yablonovitch, "Spontaneous emission extraction and Purcell enhancement from thin-film 2-D photonic crystals," J. Lightwave Technol. 17, 2096-2112 (1999).

24. T. Baba, K. Inoshita, H. Tanaka, J. Yonekura, M. Ariga, A. Matsutani, T. Miyamoto, F. Koyama, and K. Iga, "Strong enhancement of light extraction efficiency in GaInAsP 2-Darranged microcolumns," J. Lightwave Technol. 17, 21132120 (1999). 